\title{
Análises física e fisiológica de sementes de Campomanesia xanthocarpa O. Berg e Eugenia involucrata DC. (Myrtaceae) em diferentes temperaturas e substratos
}

\author{
Bruno Santiago Carvalho ${ }^{1}$, Francival Cardoso Felix ${ }^{2 *}$, Daniele Cristina Pereira de Matos ${ }^{3}$, Dagma Kratz ${ }^{4}$
}

DOI: https://doi.org/10.35699/2447-6218.2020.20458

\begin{abstract}
Resumo
Estudos voltados para os aspectos físicos de sementes e desempenho germinativo de espécies florestais nativas é de relevante interesse para tecnologistas de sementes e viveiristas. Por isso, objetivou-se avaliar a germinação de sementes de C. xanthocarpa e E. involucrata em diferentes temperaturas e substratos, bem como mensurar as características físicas e biométricas de suas sementes. Biometria, grau de umidade (GU) e peso de mil sementes (PMS) compuseram os aspectos físicos avaliados. Os testes de germinação foram conduzidos em três temperaturas (20, 25 e $30^{\circ} \mathrm{C}$ ) e três substratos (areia, vermiculita e papel mata-borrão) para C. xanthocarpa; e três temperaturas (20, 25 e $30^{\circ} \mathrm{C}$ ) e dois substratos (areia e vermiculita) para E. involucrata; avaliando-se aspectos de viabilidade e vigor de sementes. Os PMS para C. xanthocarpa e E. involucrata foram de 35,0 g (GU=25,6\%) e 471,5 g (GU=52,1\%), com 24.783 e 2.121 sementes por quilograma cada. As dimensões das sementes de C. xanthocarpa são 7,97 x 5,55 x 2,02 mm, e 12,81 x 10,02 x 7,49 mm em E. involucrata. A germinação e expressão do vigor em sementes de C. xanthocarpa e E. involucrata foram favorecidas nas temperaturas de 30 e $25^{\circ} \mathrm{C}$, limiar em que se obteve menores tempos médio de germinação (4,4 e 9,3 dias) e maiores índices de velocidade de germinação (4,8 e 1,3), com viabilidade de 99 e 76\%. Portanto, a germinação de sementes de C. xanthocarpa e E. involucrata deve ser conduzida em substrato de vermiculita e temperaturas de 30 e $25^{\circ} \mathrm{C}$, respectivamente.
\end{abstract}

Palavras-chave: Biometria. Cerejeira. Guabiroba. Germinação. Análise de sementes.

\section{Physical and physiological analysis of Campomanesia xanthocarpa O. Berg and Eugenia involucrata DC. seeds (Myrtaceae) in different temperatures and substrates}

\begin{abstract}
Studies for the physical aspects of seeds and germinative performance of native forest species are relevant for seed technologists and nurseries. The objective of this work was to evaluate the germination of C. xanthocarpa and $E$. involucrata seeds in different temperatures and substrates, and to measure the physical and biometric characteristics of the seeds. Seed biometry, water content (WC) and thousand seeds weight were the physical aspects evaluated. The germination tests were conducted at three temperatures $\left(20,25\right.$ and $30^{\circ} \mathrm{C}$ ) and three substrates (sand, vermiculite and 'mata-borrão' paper) for C. xanthocarpa; and three temperatures $\left(20,25\right.$ and $30^{\circ} \mathrm{C}$ ) and two substrates (sand and vermiculite) for E. involucrata; seed germination and vigor were evaluated. The thousand seeds weight for $C$. xanthocarpa and E. involucrata were $35.0 \mathrm{~g}(\mathrm{WC}=25.6 \%)$ and $471.5 \mathrm{~g}(\mathrm{WC}=52.1 \%)$, with 24,783 and 2,121 seeds per kilogram each. The dimensions of the $C$. xanthocarpa seeds are $7.97 \times 5.55 \times 2.02 \mathrm{~mm}$ and $12.81 \times 10.02 \times 7.49$ $\mathrm{mm}$ in $E$. involucrate seeds. The germination and expression of vigor in C. xanthocarpa and E. involucrata seeds were
\end{abstract}

\footnotetext{
${ }^{1}$ Universidade Federal do Paraná, Curitiba, Pr. Brasil.

https://orcid.org/0000-0003-3490-7762

${ }^{2}$ Universidade Federal do Paraná, Curitiba, Pr. Brasil. https://orcid.org/0000-0002-6518-5697

${ }^{3}$ Universidade Federal do Paraná, Curitiba, Pr. Brasil. https://orcid.org/0000-0001-8540-9216

${ }^{4}$ Universidade Federal do Paraná, Curitiba, Pr. Brasil. https://orcid.org/0000-0002-3062-424X

*Autor para correspondência: felixfc@outlook.com.br
}

Recebido para publicação em 06 de maio de 2020. Aceito para publicação em 08 de julho de 2020 e-ISSN: 2447-6218 / ISSN: 2447-6218. Atribuição CC BY. 
Carvalho, B. S. et al.

favored at temperatures of 30 and $25^{\circ} \mathrm{C}$, the threshold at which the lowest average germination times (4.4 and 9.3 days) and higher rates were obtained germination speed (4.8 and 1.3), with germination of 99 and $76 \%$. Therefore, the germination of $C$. xanthocarpa and E. involucrata seeds must be carried out on vermiculite substrate and temperatures of 30 and $25^{\circ} \mathrm{C}$, respectively.

Keywords: Biometry. Cerejeira. Guabiroba. Germination. Seed analysis.

\section{Introdução}

O estudo do desempenho germinativo em espécies florestais é de relevante interesse para tecnologistas de sementes e viveiristas, pois possibilita a maximização dos resultados de porcentagem, tempo médio, índice de velocidade de germinação, entre outros. Esses parâmetros estão diretamente relacionados a produtividade das mudas em viveiro e no campo, portanto, essencial para o sucesso de planos de recuperação de áreas degradadas, reflorestamentos e plantios comerciais.

A germinação de sementes envolve alterações nos aspectos bioquímicos, fisiológicos e morfológicos, os quais são desencadeados pela hidratação das células e culminam com a retomada do metabolismo e crescimento do embrião, até a formação de uma planta fotossintetizante (Marcos Filho, 2015), sendo determinada por um conjunto de fatores e condições específicas, principalmente temperatura e substratos, os quais variam para cada espécie.

A temperatura interfere diretamente na porcentagem e capacidade de germinação das sementes, bem como no mecanismo de dormência (induzido e/ ou eliminando) e na velocidade das reações bioquímicas responsáveis pela germinação (Pereira et al., 2015). A temperatura considerada ótima é aquela em que se expressa o potencial máximo de germinação e vigor da semente, temperaturas abaixo e acima do mínimo e do máximo inviabilizam a germinação (Carvalho; Nakagawa, 2012; Bewley et al., 2013; Marcos Filho, 2015).

De maneira semelhante, o substrato é um dos fatores essenciais para a germinação de sementes e desenvolvimento das plântulas, o qual pode influenciar este processo em função da sua composição, estrutura, densidade, aeração, capacidade de retenção de água e predisposição ao desenvolvimento de fitopatógenos (Oliveira et al., 2016). Os substratos mais utilizados para o teste de germinação em espécies florestais são papel (toalha, filtro e mata-borrão), areia e vermiculita (Brasil, 2013), em que sua escolha deve ser condicionada ao tamanho e a formato da semente, exigência com relação à quantidade de água, sensibilidade à luz e a facilidade que o mesmo oferece para a realização das contagens e avaliação da germinação (Brasil, 2009).

Campomanesia xanthocarpa O. Berg e Eugenia involucrata DC. são espécies florestais da família Myrtaceae, conhecidas popularmente como guabirobeira e cerejeira, respectivamente (Lorenzi, 2000). Essas espécies possuem importância na alimentação e saúde humana.
Na alimentação, ambas são utilizadas in natura ou na preparação de doces, sorvetes, geleias e licores caseiros (Vallilo et al., 2008).

O uso popular do chá das folhas de C. xanthocarpa é indicado para doenças inflamatórias, renais, digestivas e dislipidemia (Sant' Anna et al., 2017), e os extratos dos frutos de E. involucrata são uma importante fonte de antioxidantes, além das folhas serem aproveitadas na forma de chá, o qual é difundido popularmente como sendo de ação antidiarreica e digestiva (Sausen et al., 2009; Nicácio et al., 2017).

Ambas as espécies não possuem trabalhos voltados para a germinação de sementes em diferentes temperaturas e substratos, portanto, este estudo poderá contribuir para determinar em quais condições a germinação de sementes dessas espécies é mais adequada, o qual poderá subsidiar a inclusão desta metodologia nas novas publicações das Instruções para Análise de Sementes de Espécies Florestais (Brasil, 2013). Deste modo, objetivou-se avaliar o desempenho germinativo de sementes de C. xanthocarpa e E. involucrata em diferentes temperaturas e substratos, bem como mensurar as características físicas e biométricas de suas sementes.

\section{Material e métodos}

Os frutos de C. xanthocarpa e E. involucrata foram coletados de matrizes marcadas em áreas de ocorrência natural das espécies e obtidos em novembro de 2017, doação do Viveiro Chauá, localizado no município de Campo Largo, Estado do Paraná, Brasil. O beneficiamento das sementes foi feito com maceração dos frutos em peneira, secagem e extração manual das impurezas remanescentes.

Após o beneficiamento, realizou-se a caracterização biométrica das sementes de cada espécie com 50 unidades amostrais, utilizando paquímetro digital Electronic Caliper - $1112(0,01 \mathrm{~mm})$ para mensurar comprimento $(\mathrm{mm})$, largura (mm) e espessura ( $\mathrm{mm})$ das sementes. Os resultados de cada variável foram expressos em função da média, desvio padrão e coeficiente de variação (\%).

O grau de umidade das sementes foi realizado por meio da secagem em estufa a $105^{\circ} \mathrm{C} / 24 \mathrm{~h}$ (Brasil, 2009), com três repetições para cada espécie. Em seguida, determinou-se com outra amostra o peso de mil sementes conforme metodologia proposta por Brasil (2009), bem como o número de sementes por quilograma. As pesa- 
Análises física e fisiológica de sementes de Campomanesia xanthocarpa O. Berg e Eugenia involucrata DC. (Myrtaceae) em diferentes temperaturas e substratos

gens das sementes foram feitas em balança analítica de precisão Prix AS 220 R2 (0,001 g).

Para os testes de germinação, utilizaram-se germinadores do tipo Mangelsdorf sob luz constante: três temperaturas $\left(20,25\right.$ e $30^{\circ} \mathrm{C}$ ) e três substratos (areia, vermiculita e papel mata-borrão) para C. xanthocarpa; e três temperaturas $\left(20,25\right.$ e $\left.30^{\circ} \mathrm{C}\right)$ e dois substratos (areia e vermiculita) para E. involucrata. As sementes foram alocadas diretamente sobre seus respectivos substratos umedecidos em $60 \%$ da capacidade de retenção de água (Brasil, 2009), distribuídas em cinco repetições de 20 e 15 sementes para $C$. xanthocarpa e E. involucrata, respectivamente. Todos os substratos passaram por esterilização: a areia foi lavada, peneirada (malha de 0,4 $\mathrm{mm})$ e aquecida $\left(200^{\circ} \mathrm{C} / 2 \mathrm{~h}\right)$; a vermiculita foi aquecida $\left(200^{\circ} \mathrm{C} / 2 \mathrm{~h}\right)$; assim como o papel mata-borrão $\left(105^{\circ} \mathrm{C} / 2\right.$ h). Os substratos para C. xanthocarpa foram acondicionados em caixas do tipo gerbox [areia $(200 \mathrm{~g})$, vermiculita (20 g) e papel mata-borrão (duas folhas), cada], e para E. involucrata [areia (600 g) e vermiculita (60 g), cada] utilizou-se bandejas de polietileno (6,0 x 29,0 x 20,0 $\mathrm{cm}$ ), isoladas com papel filme PVC (polyvinylchloride).

Avaliaram-se as seguintes variáveis: (a) germinação - percentual de sementes germinadas (raiz primária maior que $2 \mathrm{~mm}$ ) até 20 e 28 dias após a semeadura, respectivamente, para $C$. xanthocarpa e E. involucrata; (b) índice de velocidade de germinação (IVG), (c) tempo médio de germinação (TMG) e (d) germinação acumulada - contabilização diária do número de sementes germinadas e calculado conforme fórmulas propostas por Maguire (1962), Labouriau (1983) e Labouriau e Agudo (1987), concomitantemente.

O delineamento experimental empregado foi o inteiramente ao acaso em esquema fatorial $3 \times 3$ (temperatura x substrato) para C. xanthocarpa e $3 \times 2$ (temperatura x substrato) para E. involucrata, com cinco repetições para ambas as espécies. Os dados foram submetidos ao teste de Bartlett para verificação da homogeneidade e posteriormente à análise de variância (ANOVA), quando verificada diferença significativa, realizou-se a comparação de médias pelo teste de Tukey ao nível de 5\% de probabilidade. O programa estatístico utilizado foi o Assistat 7.7 (Silva; Azevedo, 2016).

\section{Resultados e discussão}

Sementes de $C$. xanthocarpa exibiram peso de mil sementes de 35,0 g em grau de umidade de 25,6\%, contendo, portanto, 24.783 sementes por quilograma. Enquanto sementes de E. involucrata apresentaram peso de mil sementes de 471,5 g com umidade de 52,1\%, totalizando 2.121 sementes por quilograma.

Para C. xanthocarpa, valores diferentes foram encontrados para o número de sementes por quilograma (13.000 sementes) (Lorenzi, 2000), e para o peso de mil sementes $(49,0 \mathrm{~g}$ ) com 33\% de umidade (Santos et al., 2004). De maneira semelhante para E. involucrata, encontraram-se peso de mil sementes de 306,2 g sob 54,9\% de umidade (Prado, 2009), com 7.000 (Carvalho, 2009) e 7.500 sementes (Lorenzi, 2000) sementes por quilograma.

As diferenças de peso de mil sementes e número de sementes por quilograma são maximizadas em função da não padronização do grau de umidade no momento da execução dos testes, sobretudo para sementes da família Myrtaceae, as quais são recalcitrantes e podem apresentar amplas variações do conteúdo de água das sementes. Além disso, localização geográfica, coleta em diferentes indivíduos e nichos ecológicos distintos podem aumentar as diferenças na caracterização física das sementes.

Uma forma de minimizar este inconveniente, é especificando em novas publicações das Regras para Análise de Sementes (Brasil, 2009) e Instruções para Análise de Sementes Florestais (Brasil, 2013) a faixa de grau de umidade para execução do teste de peso de mil sementes, tanto para sementes ortodoxas, quanto intermediárias e recalcitrantes.

As sementes de C. xanthocarpa apresentam 7,97 \pm 0,68 mm de comprimento, 5,55 \pm 0,54 $\mathrm{mm}$ de largura e 2,02 $\pm 0,26 \mathrm{~mm}$ de espessura, ao passo que $E$. involucrata exibem $12,81 \pm 1,25 \mathrm{~mm}$ de comprimento, 10,02 $\pm 0,84 \mathrm{~mm}$ de largura e 7,49 $\pm 1,05 \mathrm{~mm}$ de espessura (Tabela 1). Ambas as espécies possuem maior dimensão na seção longitudinal, e a espessura é a variável que apresenta maior variação entre as sementes (CV).

Tabela 1 - Caracterização biométrica de sementes de Campomanesia xanthocarpa e Eugenia involucrata

\begin{tabular}{lcccccc}
\hline & \multicolumn{3}{c}{ Campomanesia xanthocarpa } & \multicolumn{3}{c}{ Eugenia involucrata } \\
\cline { 2 - 7 } Variáveis & Média & $\begin{array}{c}\text { Desvio } \\
\text { Padrão }\end{array}$ & CV (\%) & Média & $\begin{array}{c}\text { Desvio } \\
\text { Padrão }\end{array}$ & CV (\%) \\
\hline Comprimento (mm) & 7,97 & 0,68 & 8,5 & 12,81 & 1,25 & 9,8 \\
Largura (mm) & 5,55 & 0,54 & 9,7 & 10,02 & 0,84 & 8,3 \\
Espessura (mm) & 2,02 & 0,26 & 12,7 & 7,49 & 1,05 & 14,1 \\
\hline
\end{tabular}

CV: coeficiente de variação. 
Carvalho, B. S. et al.

Prado (2009) realizou a biometria em sementes de $E$. involucrata e encontrou $7,63 \pm 1,88$ de comprimento e 4,98 $\pm 1,18$ de largura, valores inferiores ao encontrado neste estudo; e Luz e Krupek (2014) encontraram valores diferentes para comprimento e largura de diásporos e peso de sementes provenientes de diferentes indivíduos de $C$. xanthocarpa. A caracterização biométrica das sementes dessas espécies é pouco difundida no meio científico e gira majoritariamente em torno do seu fruto, inviabilizando uma iminência de comparação a resultados conhecidos.

As Instruções para Análise de Sementes de Espécies Florestais constam recomendações que não foram validadas, $25-30^{\circ} \mathrm{C}$ para germinação de sementes do gênero Campomanesia em substrato vermiculita, e $25^{\circ} \mathrm{C}$ para germinação de sementes de $E$. involucrata tanto com papel ou vermiculita (Brasil, 2013).

A análise variância evidenciou que não houve interação entre os fatores temperatura e substrato nas variáveis avaliadas durante o processo germinativo para ambas as espécies, contudo, apenas o fator temperatura afetou significativamente todas as variáveis para $C$. xanthocarpa e E. involucrata, exceto germinação para $C$. xanthocarpa (Tabela 2).

Tabela 2 - Análise de variância entre os fatores temperatura e substrato para as variáveis germinação, tempo médio (TMG) e índice de velocidade de germinação (IVG) de Campomanesia xanthocarpa e Eugenia involucrata

\begin{tabular}{|c|c|c|c|c|}
\hline \multirow{2}{*}{ Fonte de variação } & \multirow{2}{*}{ GL } & \multicolumn{3}{|c|}{ QM } \\
\hline & & Germinação & TMG & IVG \\
\hline \multicolumn{5}{|l|}{ Campomanesia xanthocarpa } \\
\hline Temperatura (A) & 2 & $3,888^{\text {ns }}$ & $92,472^{* *}$ & $24,439^{* *}$ \\
\hline Substrato (B) & 2 & $3,888^{\text {ns }}$ & $0,401^{\text {ns }}$ & $0,197^{\text {ns }}$ \\
\hline Interação A x B & 4 & $3,005^{\text {ns }}$ & $0,172^{\text {ns }}$ & $0,033^{\text {ns }}$ \\
\hline Tratamento & 8 & $3,472^{\mathrm{ns}}$ & $23,304^{* *}$ & $6,176^{* *}$ \\
\hline Erro & 36 & 6,111 & 0,287 & 0,112 \\
\hline Total & 44 & & & \\
\hline CV (\%) & & 2,5 & 8,1 & 9,6 \\
\hline \multicolumn{5}{|l|}{ Eugenia involucrata } \\
\hline Temperatura (A) & 2 & $2650,369^{* *}$ & $113,171^{\text {** }}$ & $1,330^{* *}$ \\
\hline Substrato (B) & 1 & $37,037^{\mathrm{ns}}$ & $23,122^{\mathrm{ns}}$ & 0,001 ns \\
\hline Interação A x B & 2 & $19,259^{\text {ns }}$ & $24,081^{\mathrm{ns}}$ & $0,047^{\mathrm{ns}}$ \\
\hline Tratamento & 5 & $1075,259^{*}$ & $59,525^{* *}$ & $0,551^{* *}$ \\
\hline Erro & 24 & 404,444 & 7,254 & 0,117 \\
\hline Total & 29 & & & \\
\hline CV (\%) & & 31,9 & 22,4 & 38,7 \\
\hline
\end{tabular}

GL: Grau de Liberdade; QM: Quadrado Médio; CV: coeficiente de variação. ${ }^{\text {ns }}$ não significativo, "*, "significativo ao nível de 1 e 5\% de probabilidade, respectivamente.

A germinação e expressão do vigor em sementes de C. xanthocarpa e E. involucrata foram favorecidas nas temperaturas de 30 e $25^{\circ} \mathrm{C}$, respectivamente, limiar em que se obteve menores TMG (4,4 e 9,3 dias) e maiores IVG (4,8 e 1,3), com viabilidade de 99 e $76 \%$, concomitantemente (Tabela 3). Os parâmetros de tempo e velocidade de germinação refletem o vigor das sementes, uma vez que sementes vigorosas expressam todo o seu potencial de viabilidade rapidamente, e de maneira mais uniforme. Temperaturas mais elevadas favorecem a absorção de água e aceleram as reações bioquímicas das sementes, ocasionando maior atividade enzimática e mobilização de reservas durante a germinação (Bewley et al., 2013). Portanto, as temperaturas de 30 e $25^{\circ} \mathrm{C}$ em sementes de C. xanthocarpa e $E$. involucrata são favoráveis ao processo germinativo destas espécies, mutualmente.

Alta viabilidade das sementes também foi encontrada para C. xanthocarpa em substrato areia com luz artificial (93 a 100\%) e escuro (82 a 93\%), empregando diferentes temperaturas (Santos et al., 2004), ao passo que apenas $30 \%$ foram encontrados para E. involucrata em substrato rolo de papel, e $54 \%$ com o uso de vermiculita sem controle de temperatura (Prado, 2009). 
Análises física e fisiológica de sementes de Campomanesia xanthocarpa O. Berg e Eugenia involucrata DC. (Myrtaceae) em diferentes temperaturas e substratos

Herzog et al. (2012) recomendaram para germinação de sementes de C. xanthocarpa a utilização de substrato papel sem fotoperíodo a temperatura constante de $25^{\circ} \mathrm{C}$, contudo, comparam apenas substratos papel e areia. Enquanto Cripa et al. (2014) recomendam vermiculita para a germinação de E. involucrata quando comparado ao papel e areia. Esses valores se distinguem consideravelmente principalmente devido as características associadas a origem dos indivíduos, local de coleta das sementes e condições ecológicas do habitat. Outras características podem trazer influência sobre resultados distintos, como por exemplo, a caracterização física das sementes (tamanho, peso seco e grau de umidade da semente), além das condições climáticas e de disponibilidade de água durante a maturação que estabelecem relação direta com o vigor e viabilidade das sementes (Marcos Filho, 2015).

Tabela 3 - Germinação, tempo médio (TMG) e índice de velocidade de germinação (IVG) de sementes de Campomanesia xanthocarpa e Eugenia involucrata submetidas ao processo germinativo em diferentes temperaturas

\begin{tabular}{cccc}
\hline \multirow{2}{*}{ Temperaturas $\left({ }^{\circ} \mathrm{C}\right)$} & Germinação (\%) & TMG (dias) & IVG \\
\cline { 2 - 4 } & $99 \mathrm{a}$ & Campomanesia xanthocarpa \\
\hline 20 & $99 \mathrm{a}$ & $9,3 \mathrm{a}$ & $2,3 \mathrm{c}$ \\
25 & $99 \mathrm{a}$ & $6,2 \mathrm{~b}$ & $3,4 \mathrm{~b}$ \\
30 & & $4,4 \mathrm{c}$ & $4,8 \mathrm{a}$ \\
\hline & $68 \mathrm{a}$ & Eugenia involucrata \\
\hline 20 & $76 \mathrm{a}$ & $15,8 \mathrm{a}$ & $0,7 \mathrm{~b}$ \\
30 & $45 \mathrm{~b}$ & $9,3 \mathrm{~b}$ & $1,3 \mathrm{a}$ \\
\end{tabular}

Médias seguidas da mesma letra não diferem estatisticamente pelo teste de Tukey ao nível de 5\% de probabilidade.

A germinação e expressão do vigor em sementes de C. xanthocarpa e E. involucrata não foram afetadas em função do tipo de substrato utilizado, obtendo-se viabilidade média de 99 e 64\%, respectivamente (Tabela 4). Deste modo, a utilização por qualquer substrato testado pode ser feita para a germinação das sementes destas espécies, pois apresentarão a mesma eficácia. Contudo, em caso de aproveitamento do material genético para produção de mudas, recomenda-se a instalação dos testes de germinação em substrato vermiculita ou areia, a fim de mantê-las até a obtenção de plântulas para realização da repicagem.

Tabela 4 - Germinação, tempo médio (TMG) e índice de velocidade de germinação (IVG) de sementes de Campomanesia xanthocarpa e Eugenia involucrata submetidas ao processo germinativo em diferentes substratos

\begin{tabular}{cccc}
\hline \multirow{2}{*}{ Substratos } & Germinação (\%) & TMG (dias) & IVG \\
\cline { 2 - 4 } & & Campomanesia xanthocarpa \\
\hline Areia & $99 \mathrm{a}$ & $6,8 \mathrm{a}$ & $3,4 \mathrm{a}$ \\
Vermiculita & $99 \mathrm{a}$ & $6,5 \mathrm{a}$ & $3,5 \mathrm{a}$ \\
Papel & $99 \mathrm{a}$ & $6,5 \mathrm{a}$ & $3,6 \mathrm{a}$ \\
\hline & & Eugenia involucrata \\
\hline Areia & $64 \mathrm{a}$ & $12,9 \mathrm{a}$ & $0,9 \mathrm{a}$ \\
Vermiculita & $62 \mathrm{a}$ & $11,2 \mathrm{a}$ & $0,9 \mathrm{a}$ \\
\hline
\end{tabular}

Médias seguidas da mesma letra não diferem estatisticamente pelo teste de Tukey ao nível de 5\% de probabilidade.

A vermiculita é mais indicada para sementes maiores porque mantém boa umidade e aeração do substrato, minimizando a proliferação de fungos, enquanto o substrato areia é menos recomendado em decorrência da necessidade de lavagem, homogeneização e cuidados com o transporte, o qual é mais difícil de operar devido ao peso dos recipientes e pode causar possíveis dados ao sistema radicial das plântulas. Portanto, torna-se cada vez mais rotineiro o uso da vermiculita para germinação de sementes de espécies florestais (Brasil, 2013; Pereira et al., 2015; Oliveira et al., 2016).

A germinação acumulada é outro parâmetro que nos permite escolher a temperatura e substrato mais adequados para a germinação das sementes de uma espécie. Para C. xanthocarpa e E. involucrata, observa-se diferentes 
Carvalho, B. S. et al.

padrões de germinação em função das temperaturas e substratos empregados (Figuras 1A e 1B). Predomina para C. xanthocarpa maior uniformidade e estabilização da germinação a $30^{\circ} \mathrm{C}$, independentemente do substrato utilizado, e para E. involucrata, $25^{\circ} \mathrm{C}$ é a temperatura em que ocorre maior uniformidade com o uso dos substratos areia e vermiculita.

Figura 1 - Germinação acumulada de sementes de Campomanesia xanthocarpa (A) e Eugenia involucrata (B) submetidas ao processo germinativo sob diferentes temperaturas $\left(20,25\right.$ e $\left.30^{\circ} \mathrm{C}\right)$ e substratos $(\mathrm{V}=\mathrm{Vermiculita}$; $\mathrm{A}=$ Areia; $\mathrm{PMB}=$ Papel mata-borrão)
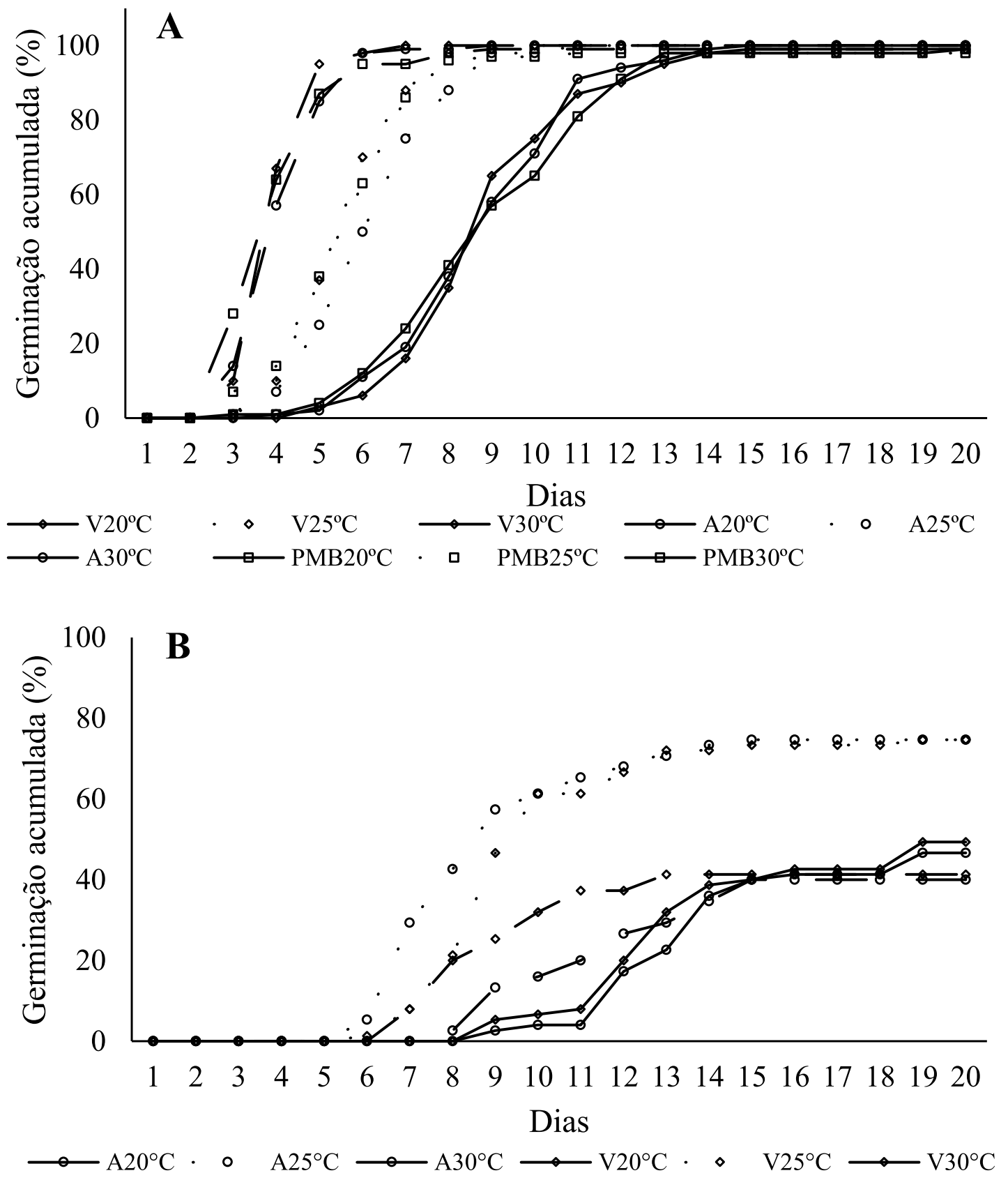

\section{Conclusão}

A germinação de sementes de C. xanthocarpa e $E$. involucrata (Myrtaceae) deve ser conduzida em substrato de vermiculita e temperaturas de 30 e $25^{\circ} \mathrm{C}$, respectiva- mente. E, as análises dos aspectos físicos contribuíram para a caracterização física e biométrica das sementes destas espécies. 


\section{Referências}

Bewley, J. D.; Bradford, K. J.; Bilhorst, H. W. M.; Nonogaki, H. 2013. Seeds physiology of development, germination and dormancy. 3. ed. New York: Springer.

Brasil. Ministério da Agricultura, Pecuária e Abastecimento. 2009. Regras para análise de sementes. Brasília: Secretaria de Defesa Agropecuária. MAPA/ACS. Disponível em: https://cutt.ly/EyjBvi0.

Brasil. Ministério da Agricultura, Pecuária e Abastecimento. 2013. Instruções para a análise de sementes de espécies florestais. Brasília: MAPA/ACS. Disponível em: https://cutt.ly/tyjBnF6.

Carvalho P. E. R. 2009. Cerejeira Eugenia involucrata. Colombo: EMBRAPA (Comunicado Técnico, 224). Disponível em: https://cutt. ly/WyjBmV7.

Carvalho, N. M.; Nakagawa, J. 2012. Sementes: ciência, tecnologia e produção. 5. ed. Jaboticabal: FUNEP.

Cripa, F. B.; Freitas, L. C. N.; Grings, A. C.; Bortolini, M. F. 2014. Tetrazolium test for viability e stimation of Eugenia involucrata DC. and Eugenia pyriformis Cambess. seeds. Journal of Seed Science, 36: 305-311. Doi: https://doi.org/10.1590/2317-1545v36n3991.

Herzog, N. F. M.; Malavasi, M. M.; Malavasi, U. C. 2012. Fruit morfometry and germination of Campomanesia xanthocarpa O. Berg seeds. Semina: Ciências Agrárias, 33: 1359-1366. Doi: http://dx.doi. org/10.5433/1679-0359.2012v33n4p1359.

Labouriau, L. F. G. 1983. Germinação das sementes. Washington: Secretaria da OEA.

Laboriau, L. G.; Agudo, M. 1987. On the physiology of seed germination in Salvia hispanica L. I. temperature effects. Anais da Academia Brasileira de Ciências, 59: 37-56.

Lorenzi, H. 2000. Árvores Brasileiras. 5. ed. Nova Odessa: Instituto Plantarum de estudos da flora.

Luz, I. J.; Krupek, R. A. 2014. Fenologia reprodutiva, biometria do fruto e semente de Campomanesia xanthocarpa O. Berg. (Myrtaceae). Estudos de Biologia, 36: 115-124. Doi: http://dx.doi.org/10.7213/ estud.biol.36.086.AO12.

Maguire, J. D. 1962. Speed of germination aid seedling emergence and vigor. Crop Science, 2: 176-177.
Marcos Filho, J. 2015. Fisiologia de sementes de plantas cultivadas. 2. ed. Londrina: ABRATES.

Nicácio, A. E.; Rotta, E. M.; Boeing, J. S.; Barizão, E. O.; Kimura, E.; Visentainer, J. V.; Maldaner, L. 2017. Antioxidant activity and determination of phenolic compounds from Eugenia involucrata DC. fruits by UHPLC-MS/MS. Food Analytical Methods, 10: 2718-2728. Doi: https://doi.org/10.1007/s12161-017-0840-3.

Oliveira, F. N.; França, F. D.; Torres, B. T.; Nogueira, N. W.; Freitas, R. M. O. 2016. Temperaturas e substratos na germinação de sementes de pereiro-vermelho (Simira gardneriana M.R. Barbosa \& Peixoto). Revista Ciência Agronômica, 47: 658-666. Disponível em: https:// cutt.ly/yyjBTNH.

Pereira, K. T. O.; Santos, B. R. V.; Benedito, C. P.; Lopes, E. G.; Aquino. G. S. M. 2015. Germinação e vigor de sementes de Moringa oleifera Lam. em diferentes substratos e temperaturas. Revista Caatinga, 28: 92-99. Disponível em: https://cutt.ly/wyjBUg4.

Prado A. P. 2009. Aspectos autoecológicos e silviculturais de Eugenia involucrata DC. Santa Maria: Universidade Federal de Santa Maria, 134 f. Dissertação de Mestrado. Disponível em: https://cutt.ly/YyjBI3S.

Sant' Anna, L. S.; Merlugo, L.; Ehle, C. S.; Limberger, J.; Fernandes, M. B.; Santos M. C.; Mendez, A. S.; Paula, F. R.; Moreira, C. M. 2017. Chemical composition and hypotensive effect of Campomanesia xanthocarpa. Evidence-Based Complementary and Alternative Medicine. 2017: 1-11. Doi: https://doi.org/10.1155/2017/1591762.

Santos, C. M. R.; Ferreira, A. G.; Áquila, M. E. A. 2004. Características de frutos e germinação de sementes de seis espécies de Myrtaceae nativas do Rio Grande do Sul. Ciência Florestal, 12: 13-20. Doi: https://doi. org/10.5902/198050981802.

Sausen, T. L.; Löwe, T. R.; Figueiredo, L. S.; Buzatto, C. R. 2009. Avaliação da atividade alelopática do extrato aquoso de folhas de $E$. involucrata e A. sellowiana. Polibotânica, 27: 145-158. Disponível em: https://cutt.ly/jyjBSUz.

Silva, F. A. S.; Azevedo, C. A. V. 2016. The Assistat software version 7.7 and its use in the analysis of experimental data. African Journal of Agricultural Research, 11: 3733-3740. Doi: https://doi.org/10.5897/ AJAR2016.11522.

Vallilo, M. I.; Moreno, P. R. H.; Oliveira, E.; Lamardo, L. C. A.; Garbelotti, M. L. 2008. Chemical composition of Campomanesia xanthocarpa Berg -Myrtaceae fruit. Food Science and Technology, 28: 231-237. Doi: https://doi.org/10.1590/S0101-20612008000500035. 